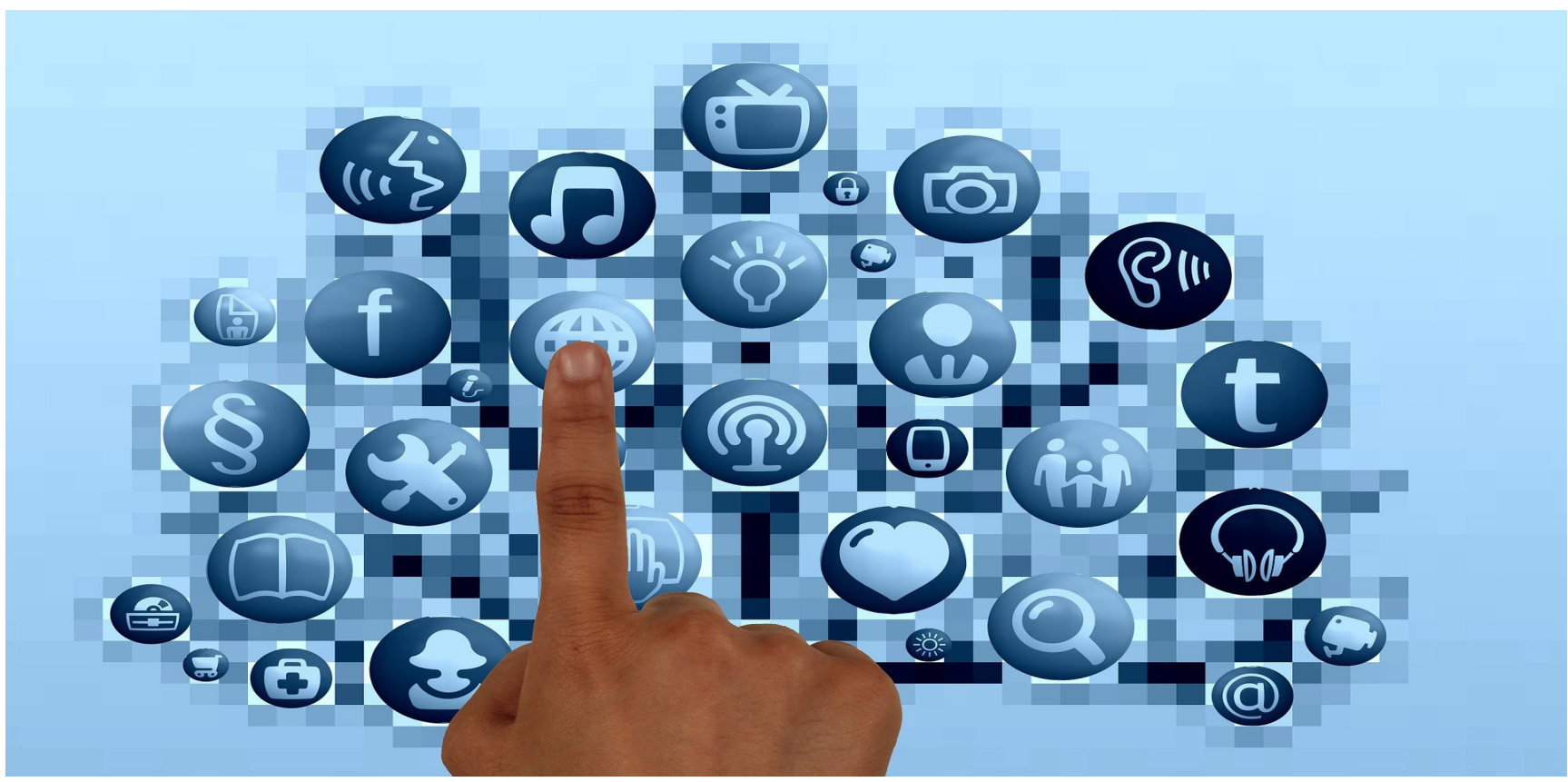

\title{
Comercio electrónico en el sector artesanal en Norte de Santander
}

\section{E-commerce in the handicraft sector in Norte de Santander}

\section{Lina Andrea Cortés-Miranda}

Estudiante, Fundación de Estudios Superiores Comfanorte, Cúcuta, Colombia

Angie Paola Galeano-Solano

Estudiante, Fundación de Estudios Superiores Comfanorte, Cúcuta, Colombia

Saray Daniela Nader-Sánchez

Estudiante, Fundación de Estudios Superiores Comfanorte, Cúcuta, Colombia
Karen Dayana Saboyá-García

Estudiante, Fundación de Estudios Superiores Comfanorte, Cúcuta, Colombia

Ender José Barrientos-Monsalve Doctor en Ciencias Gerenciales, ej_barrientos@fesc.edu.co, Fundación de Estudios Superiores Comfanorte, Cúcuta, Colombia. 
Cómo citar: Camacho-Castillo, A.M., Torres-Mendoza, D.M., Barrientos-Monsalve, E.J. (2020). Gestión de intangibles en la industria informal de la moda en el subsector confecciones de Cúcuta. Reflexiones contables (Cúcuta), 3 (1), 51-56.

\section{Resumen}

El artículo está enfocado en conocer si el comercio electrónico está siendo aprovechado como herramienta que impulse la compra y venta de artículos artesanales en Norte de Santander. Precisamente, el avance de estos ha planteado nuevos horizontes en diferentes aspectos del comercio electrónico, como lo es la venta y compra de productos de una forma más rápida y fácil tanto para el consumidor como para el vendedor. El comercio electrónico ha experimentado una notable evolución, pasando de ser un simple catálogo de productos o servicios, construido a partir de una página estática, a convertirse en un medio eficaz para realizar negocios. El comercio electrónico es una herramienta que si se aprovecha puede traer los mejores beneficios para las empresas.

Palabras clave: beneficio, comercio electrónico, evolución, página web, productos, servicios.

\section{Abstract}

The article focuses on whether e-commerce is being used as a tool to promote the purchase and sale of handicrafts in Norte de Santander. Precisely, the advance of these has raised new horizons in different aspects of e-commerce, such as the sale and purchase of products in a faster and easier way for both the consumer and the seller. E-commerce has undergone a remarkable evolution from being a simple catalogue of products or services, built from a static page, to becoming an effective means of doing business. E-commerce is a tool that, if harnessed, can bring the greatest benefits to companies.

Keywords: profit, e-commerce, evolution, website, products, services. 


\section{Introducción}

El comercio electrónico, o e-commerce, es la compra-venta de productos o servicios a través de Internet y redes informáticas. Además, conlleva tareas como el marketing, contenidos e información en la red. Todo ello supone una revolución en la manera en la que las empresas se comunican y desarrollan su actividad comercial. La importancia de este método es la accesibilidad que los clientes de una empresa pueden tener a los productos o servicios que ofrece la empresa, desde cualquier lugar y en cualquier momento (Cegos, 2020).

Según cifras del Observatorio de Ecommerce (2020) Europa $(\$ 482.3 \mathrm{bn})$ y Norteamérica (\$452.4bn) se encuentran entre las primeras regiones donde más facturación se genera. Sin embargo, en la actualidad, quien tiene un mayor crecimiento es Asia-Pacifico (un 44.5\%) con una facturación en el 2013 de 567.3bn de dólares. De igual forma Latinoamérica muestra una gran evolución, con un crecimiento del $21.5 \%$ y una facturación de $\$ 33.2 \mathrm{bn}$. Los países de esta región que hoy más compran por Internet son: Brasil, Venezuela, Argentina, Colombia, México, Perú y Chile. ComScore afirma que ocho de cada diez consumidores latinoamericanos buscan, investigan y compran productos en línea semanalmente.

Actualmente el comercio electrónico ha experimentado un fuerte crecimiento a nivel global, tanto en volumen de usuarios como de sitios comerciales; sin duda alguna la inversión en publicidad ha contribuido a su acelerado desarrollo de una manera exponencial. Hoy es la manera fácil y rápida de hacer negocios sin necesidad de grandes inversiones, desde la comodidad de su hogar u oficina, pues solo se requiere un computador y acceso a Internet. (Troconis, 2019). Es innegable la importancia que el Internet ha adquirido en la cotidianidad de las personas, gracias a la inmediatez y comodidad que brinda. Hacer mercado, comprar regalos, transferir dinero y hasta pagar los servicios básicos del hogar se ha vuelto mucho más sencillo pues esta herramienta brinda la posibilidad de hacerlo todo desde un dispositivo sin gastar tiempo en filas ni desplazamientos. (Portafolio, 2013) Colombia representa hoy en día, una gran oportunidad para el comercio electrónico. La política del gobierno colombiano que incluye masificación y apropiación de tecnologías digitales, el crecimiento económico y los cambios en los hábitos de compra del consumidor colombiano donde Internet juega un rol cada vez más importante (Paiño, 2014). El país cuenta con el $63 \%$ de compras en línea, esto da a exponer que es una alta cifra de compradores. Dentro del país existen diferentes páginas web en donde se pueden adquirir diferentes tipos de artículos, como Ebay, Mercado libre, Amazon, OLX, Poppe, entre otras. (Observatorio de Ecommerce, 2019), permitiendo su entrada a una etapa de ascenso donde el comercio electró $n$ nico no va a parar de crecer. Aunque se trata de un fenómeno panregional, Colombia tiene una serie de particularidades que lo hacen, hoy por hoy, uno de los mercados más atractivos para desarrollar negocios electrónicos y sobre el cual han puesto su mira grandes jugadores locales e internaciona $\neg$ les (Soler, 2016)

Según el estudio Global de Nielsen sobre Comercio Conectado, el 40 por ciento de los colombianos ha comprado alguna vez ropa o productos de moda en páginas web, mientras que el 39 por ciento ha adquirido dispositivos electrónicos por este medio, comprobando su aceptación y participación en el mercado digital (Gutiérrez Tobar, 2015)

Según el más reciente estudio de la Cámara Colombiana de Comercio Electrónico (2019), en nuestro país el $76 \%$ de los internautas han hecho compras por internet al menos una vez en su vida, y desde el 2016, las compras por internet anualmente oscilan alrededor de los 26000 dólares, cifra considerablemente positiva, pues representa un crecimiento de 


\section{4}

más del $60 \%$ en los últimos dos años. Este fenómeno ha sido posible gracias a que cada vez son más los comerciantes que se atreven a migrar a las plataformas digitales y lazar sus productos en este mercado, ya sea de manera exclusiva o como un complemento a las tiendas físicas en formato tradicional.

Con el pasar de los años y la evolución de la tecnología, Colombia ha sido uno de los países que se ha ido adaptando a estos cambios para poder aplicarlos en el día a día, si bien, el comercio electrónico ha sido una de las mayores ventajas en el mundo, las personas lo han adoptado como una nueva forma de trabajo, sin embargo, no todo el País ha sabido aprovecharlo (Roa Zapata \& Cuellar Bautista, 2019). Para los artistas como diseñadores, artesanos, modistas, entre otros, vender sus artículos no siempre es fácil. Si bien, en Colombia se pueden encontrar páginas como Etsy, Ebay, Mercado libre, entre otros, sin embargo, no cumplen con las características necesarias para considerarlas como una tienda electrónica diseñada para los artesanos y trabajadores independientes que quieran vender sus artículos, sumándole el hecho de que la moneda no siempre es la más asequible tanto para el cliente como para el vendedor, al igual que el tiempo de los envíos (Artesanías de Colombia, 2017).

El objetivo principal de este artículo es conocer el uso del comercio electrónico como un impulso para la compra y venta de artículos artesanales en Norte de Santander, ya que en el departamento no se ha aprovechado totalmente los beneficios que este trae tanto para los trabajadores independientes como para las empresas que realizan trabajos artesanales. La presente investigación también se enfocará en identificar cuáles son las necesidades de los artistas independientes en el departamento de Norte de Santander, ya que, existen muchas personas que elaboran productos a mano como accesorios, artesanías, ropa, manualidades, arte digital, entre otros, y a veces no encuentran el medio adecuado para poder comercializarlos, por lo tanto, el siguiente trabajo busca indagar y conocer aquellas estrategias que se pueden implementar para cumplir con las necesidades de estos emprendedores, para que puedan comercializar su trabajo de una forma segura, rápida y eficaz usando los beneficios que las tecnologías nos está brindando hoy en día.

\section{Materiales y Métodos}

La investigación es diseño descriptivo, con enfoque cuantitativo, se aplicó como instrumento una encuesta dirigida a 20 personas con conocimientos básicos en la compra electrónica de productos.

\section{Resultados y análisis}

El $40 \%$ de la población encuestada votó por el pago contra entrega, mientras que un $30 \%$ prefiere hacer los pagos por Efecty, por otro lado el $20 \%$ paga con tarjeta de crédito y un $10 \%$ de la población por la aplicación Nequi (gráfica 1)

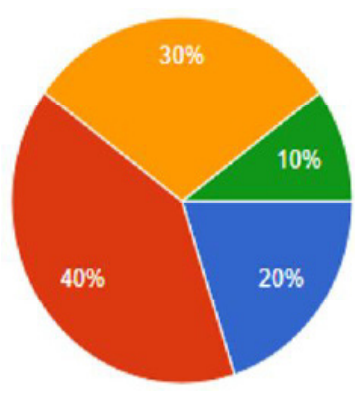

Tarjeta crédito

Pago contraentrega Efecty

Nequi

Gráfica 1. ¿Qué formas de pago utiliza al comprar en una página web?

Como se puede observar en la gráfica 2, la mayoría de la población encuestada con un $90 \%$ no tiene conocimiento de alguna página web en Norte de Santander. 


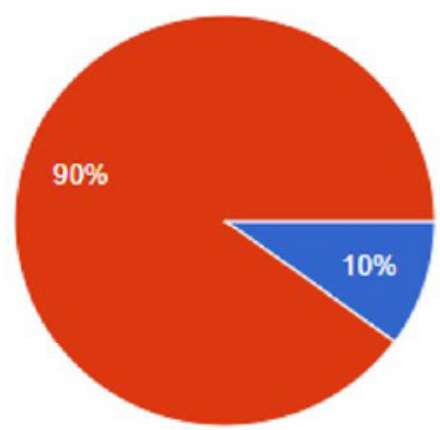

Gráfica 2 ¿Conoce alguna página de venta de artículos vintage en Norte de Santander?

Todas las personas encuestadas si creen que hace falta páginas web que vendan artículos artesanales en Norte de Santander (gráfica 3)

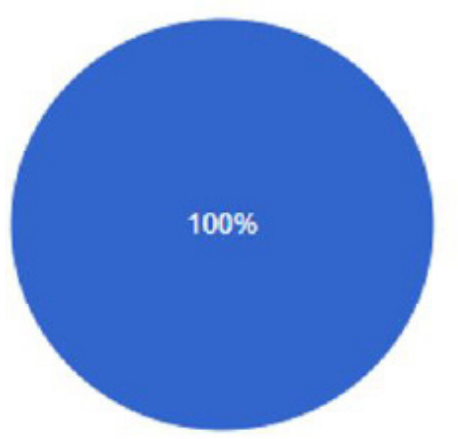

Gráfica 3. ¿Cree usted que hacen falta páginas web que vendan este tipo de artículos en Norte de Santander?

El 90\% respondió que no ha comprado artículos en páginas web en Norte de Santander, esto por la carencia de las mismas, mientras que un $10 \%$ sí ha comprado (gráfica 5).

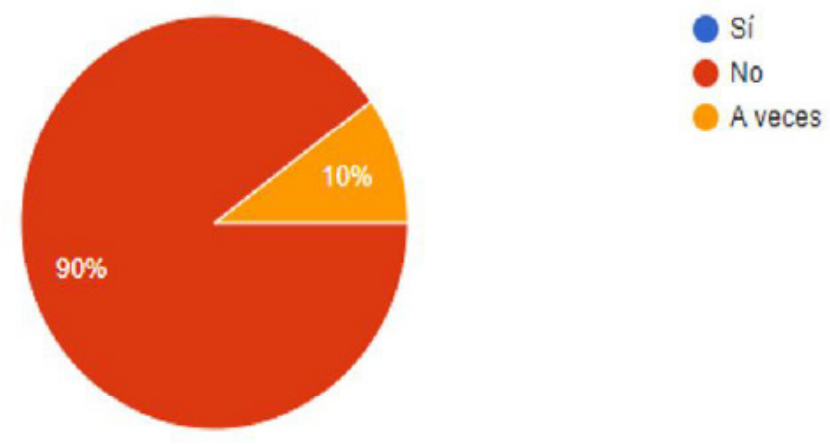

Gráfica 5. ¿Ha comprado usted artículos vintage por medio de páginas web en Norte de Santander?

\section{Conclusiones}

El mercado electrónico es bastante amplio en Colombia, sin embargo, no existe una plataforma digital para la venta y compra de artículos artesanales en el departamento de Norte de Santander, a esto se le suma la falta de medios para vender el trabajo de los artistas independientes del departamento, el pago en otras monedas en páginas exteriores del país y la demora en los envíos de los productos.

Es importante que la población del país y, sobre todo, Norte de Santander se pueda adaptar correctamente a los cambios tecnológicos para aprovechar las ventajas que estos traen consigo, abarcándolo desde todos los ámbitos.

En el Departamento de Norte de Santander ya que el medio siempre es competitivo y no existen páginas donde los artesanos puedan tener un espacio exclusivo para vender sus productos, un espacio donde las personas puedan llegar directamente al objetivo de lo que están buscando.

\section{Referencias}

Artesanías de Colombia. (2017). Diagnóstico del sector artesanal en Colombia. Disponible en: https://repositorio.artesaniasdecolombia.com. co/bitstream/001/4102/1/INST-D\%202017.\%20 41.pdf

Buenaño, D. A., Bustillos, H. M., \& Moreno, G. G. (2014). Comunicación 360: herramientas para la gestión de comunicación interna e identidad. Facultad de Comunicación-Universidad Tecnológica Equinoccial.

Cámara Colombiana de Comercio Electrónico. (2019). El eCommerce continúa creciendo en Colombia y aumentan las visitas a través de dispositivos móviles. Disponible en: https:// www.ccce.org.co/noticias/el-ecommercecontinua-creciendo-en-colombia-y-aumentan- 


\section{6}

las-visitas-a-traves-de-dispositivos-moviles/

Cegos. (2020). ¿Qué es el comercio electrónico? Disponible en: https://www. cegosonlineuniversity.com/que-es-elcomercio-electronico/

Gutiérrez Tobar, E. (2015). Impacto del comercio electrónico en las grandes y medianas empresas de la ciudad de Sogamoso Boyacá, Colombia, Cuadernos Latinoamericanos de Administración, XI(20), 83-96

Observatorio de Eco-mmerce (2020). Cómo crece el Comercio Electrónico en el mundo. Disponible en: http://observatorioecommerce. com/como-crece-el-comercio-electronico-enel-mundo/

Observatorio de Ecommerce. (2019). Medición de Indicadores de consumo del Observatorio eCommerce. Disponible en: https://www. observatorioecom merce.com.co/w p content/uploads/2019/03/estudio-consumoecommerce-colombia-observatorio-2019.pdf

Portafolio (2013). Compras online: ¿cuáles son sus ventajas? Disponible en: https://www. portafolio.co/tendencias/10-ventajas-comprasinternet-75878

Roa Zapata, E. F., \& Cuellar Bautista, D. (2019). Evolución del comercio electrónico en Colombia en la última década. Retrieved from https://ciencia.lasalle.edu.co/administracion de_empresas $/ 3080$

Soler Patiño, A. (2016). ¿Hacia dónde va el comercio electrónico en Colombia?. Ploutos, 4(1), 17-24. Recuperado a partir de https:// journal.universidadean.edu.co/index.php/plou/ article/view/1371 